\title{
Simultaneous Measurement of Multiple Independent Atomic-Scale Interactions Using Scanning Probe Microscopy: Data Interpretation and the Effect of Cross-Talk
}

Mehmet Z. Baykara, ${ }^{*}, \dagger, \ldots$ Milica Todorović, ${ }^{\perp}$ Harry Mönig, ${ }^{\dagger, \ddagger}, \#$ Todd C. Schwendemann, ${ }^{\dagger, \ddagger}, \nabla$ Lucia Rodrigo, ${ }^{\perp}$ Eric I. Altman, ${ }^{\ddagger}$, Rubén Pérez, $^{\perp, O}$ and Udo D. Schwarz ${ }^{*, \dagger, \ddagger, \S}$

${ }^{\dagger}$ Department of Mechanical Engineering and Materials Science, ${ }^{\ddagger}$ Center for Research on Interface Structures and Phenomena (CRISP), and ${ }^{\S}$ Department of Chemical and Environmental Engineering, Yale University, New Haven, Connecticut 06520, United States

"Department of Mechanical Engineering and UNAM-Institute of Materials Science and Nanotechnology, Bilkent University, 06800 Ankara, Turkey

${ }^{\perp}$ Departamento de Física Teórica de la Materia Condensada, Universidad Autónoma de Madrid, 28049 Madrid, Spain

\#Institute of Physics and Center for Nanotechnology (CeNTech), University of Münster, 48149 Münster, Germany

${ }^{\nabla}$ Physics Department, Southern Connecticut State University, New Haven, Connecticut 06515, United States

${ }^{\circ}$ Condensed Matter Physics Center (IFIMAC), Universidad Autónoma de Madrid, 28049 Madrid, Spain

ABSTRACT: In high-resolution scanning probe microscopy, it is becoming increasingly common to simultaneously record multiple channels representing different tip-sample interactions to collect complementary information about the sample surface. A popular choice involves simultaneous scanning tunneling microscopy (STM) and noncontact atomic force microscopy (NC-AFM) measurements, which are thought to reflect the chemical and electronic properties of the sample surface. With surface-oxidized $\mathrm{Cu}(100)$ as an example, we investigate whether atomic-scale information on chemical interactions can be reliably extracted from frequency shift maps obtained while using the tunneling current as the feedback parameter. Ab initio calculations of interaction forces between specific tip apexes and the surface are utilized to compare experiments with theoretical expectations. The examination reveals that constant-current operation may induce a noticeable influence of topography-feedback-induced cross-talk on the frequency shift data, resulting in misleading interpretations of local chemical interactions on the surface. Consequently, the need to apply methods such as 3D-AFM is emphasized when accurate conclusions about both the local charge density near the Fermi level, as provided by the STM channel, and the sitespecific strength of tip-sample interactions (NC-AFM channel) are desired. We conclude by generalizing to the case where multiple atomic-scale interactions are being probed while only one of them is kept constant.

\section{INTRODUCTION}

With the accelerating trend toward miniaturization in functional electromechanical systems as well as the advent of twodimensional materials with exceptional physical properties such as graphene ${ }^{1}$ and silica bilayers, ${ }^{2}$ measuring and understanding chemical surface forces with atomic specificity has become increasingly important for fields as diverse as molecular electronics, catalysis, and tribology. ${ }^{3}$ To image and characterize surfaces with atomic resolution, scanning tunneling microscopy $(\mathrm{STM})^{4}$ and noncontact atomic force microscopy (NC-AFM) ${ }^{5}$ are most frequently used. While STM relies on the tunneling current between an atomically sharp probe tip and a (semi-) conducting sample to deliver atomic scale information about the electronic properties of surfaces, NC-AFM maps how much the resonance frequency $f$ of an oscillating cantilever has changed from its value away from the surface (i.e., from its eigenfrequency $f_{0}$ ) due to the chemical interaction forces acting between the tip and the sample (corresponding to the frequency shift, $\left.\Delta f=f-f_{0}\right)$. Consequently, the concurrent recording of both the STM and the NC-AFM channel has in principle the potential to yield complementary information on both atomic scale variations in surface interactions and the underlying electronic structure responsible for them. However, using a combination of atomic-scale topography data acquired with tunneling current-based feedback and simultaneously recorded frequency shift images on surface oxidized $\mathrm{Cu}(100)$, we show in this paper that topography-feedback-induced cross-talk between the two channels leads to complications in the correct

Received: January 20, 2015

Revised: March 4, 2015

Published: March 5, 2015 
a)

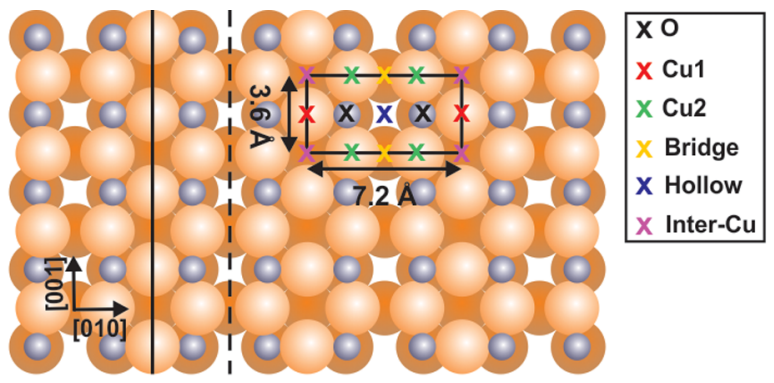

b)

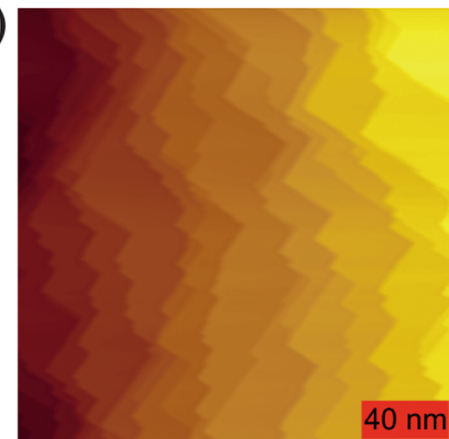

Figure 1. (a) Model of the $(2 \sqrt{ } 2 \times \sqrt{ } 2) R 45^{\circ}$ missing row reconstruction associated with the surface oxide layer on $\mathrm{Cu}(100)$. Surface $\mathrm{Cu}$ atoms are displayed as light orange spheres, second layer $\mathrm{Cu}$ atoms as dark orange spheres, and surface oxygen atoms as small gray spheres. Color-coded crosses in the unit cell denote the six lattice sites for which tip-sample force interactions have been calculated via ab initio methods. Oxygen atoms (O) are marked by black crosses, whereas copper atoms at the center of filled rows (Cu1) are marked by red and copper atoms at the edges of missing rows $(\mathrm{Cu} 2)$ by green crosses. Similarly, yellow crosses indicate the locations of the bridge sites between two Cu2 atoms on the missing rows while the hollow sites between two $\mathrm{O}$ atoms on the missing rows are highlighted by blue crosses. Finally, pink crosses denote the lattice sites surrounded by four $\mathrm{Cu}$ atoms in the filled rows (Inter-Cu). (b) Large-scale STM image of the surface structure $(U=0.20 \mathrm{~V}, I=1.0 \mathrm{nA}, T=60 \mathrm{~K})$. Atomically flat terraces are separated by steps pinned predominantly in the $\langle 001\rangle$ directions of the substrate.

assignment of spatial variations in $\Delta f$ to individual atomic locations.

It has been demonstrated before that quantification of the local chemical interaction forces can be accomplished via the method of dynamic force spectroscopy (DFS) by using the frequency shift data recorded in NC-AFM images. ${ }^{6-8}$ The technique has been extended in recent years to three spatial dimensions above the sample surface via the method of threedimensional atomic force microscopy (3D-AFM). ${ }^{9}$ Using 3DAFM, several impressive accomplishments in atomic-scale characterization of surface properties have been reported, including (i) the quantification of the chemical interaction forces on a graphite surface with very high spatial resolution to gain information about associated frictional properties, ${ }^{10,11}$ (ii) the high resolution measurement of chemical interaction forces on a single organic molecule, ${ }^{12}$ and (iii) the detailed investigation of phenomena of fundamental importance such as hydrogen bonding. ${ }^{13}$ Moreover, the combination of the $3 \mathrm{D}$ AFM method with simultaneously performed STM has allowed the complementary acquisition of atomic-scale chemical and electronic information on material surfaces, facilitating interpretation of contrast formation mechanisms and issues such as bond symmetry and atomic-scale defect identification. $^{14,15}$

While combined 3D-AFM/STM can provide a wealth of information regarding atomic-scale surface properties, its application is far from routine, as the method requires extended measurement times due to the acquisition of multiple images or spectroscopy curves as well as several postmeasurement data processing steps. ${ }^{16,17}$ In an attempt to obtain information on both chemical interactions and surface charge distributions with less experimental effort, several research groups have acquired simultaneous two-dimensional STM/NC-AFM data instead. Three approaches have been used. In mode (i), the surface is imaged using NC-AFM feedback while the average tunneling current during oscillation $I_{\text {avg }}$ is collected at the same time. ${ }^{18,19}$ Mode (ii) uses STM feedback, but oscillates the cantilever, which allows simultaneous collection of the frequency shift $\Delta f .^{19-22}$ This mode is usually referred to as the dynamic STM mode. Finally, mode (iii) involves imaging the surface while holding the tip at a constant (or almost constant) height and recording the tunneling current and the frequency shift as a function of lateral position. ${ }^{23-25}$

With the premise of getting reliable information on local interaction forces and currents, mode (iii) is ideally suited, as due to the constant-height operation there is no cross-talk between the separate data channels, which may otherwise occur due to the feedback-induced vertical motion of the tip above the surface. It has indeed been demonstrated that mode (iii) can be utilized toward, for example, the discrimination of surface vacancies from adsorbed molecules on semiconductor surfaces. ${ }^{26}$ On many extended surfaces, however, steps and/or irregularities and defects, such as structural domain boundaries and adsorbates, result in an increased likelihood of tip instabilities and even crashes with the surface when operating without topographic feedback control, complicating such measurements. Moreover, even if small, perfect terraces could be imaged without incident, it is exactly the role of these defects in governing the macroscopic chemical properties of the surface that is often of greatest interest. ${ }^{27}$ Therefore, most practical applications employ either mode (i) or (ii).

In this paper, we examine simultaneous STM/NC-AFM measurements performed on the surface oxide layer of $\mathrm{Cu}(100)$ using mode (ii) (the dynamic STM mode) with a special focus on answering the following question: Is it possible to gain a qualitative yet precise understanding of atomic-scale chemical interaction forces on sample surfaces from $\Delta f$ data acquired during such measurements? Answering this question is relevant since the dynamic STM mode is predominantly chosen in combined STM/NC-AFM measurements due to its operational robustness compared with mode (i). The surface oxide layer on $\mathrm{Cu}(100)$ provides a well-studied model system where the various contrasts observed in the STM channel have been previously associated with specific tip apex structures, ${ }^{15,28}$ which in turn allows a comparison of the simultaneously recorded $\Delta f$ values with ab initio calculations of force interactions. Moreover, scanning probe microscopy (SPM) measurements have contributed significantly to a detailed understanding of atomic-scale surface chemistry in relation to processes such as heterogeneous catalysis. ${ }^{9,29}$ Particular areas where SPM measurements have already delivered remarkable results are the determination of adsorption sites ${ }^{30}$ as well as metal-support interactions. ${ }^{31}$ Catalysts based on copper 
a)

c)

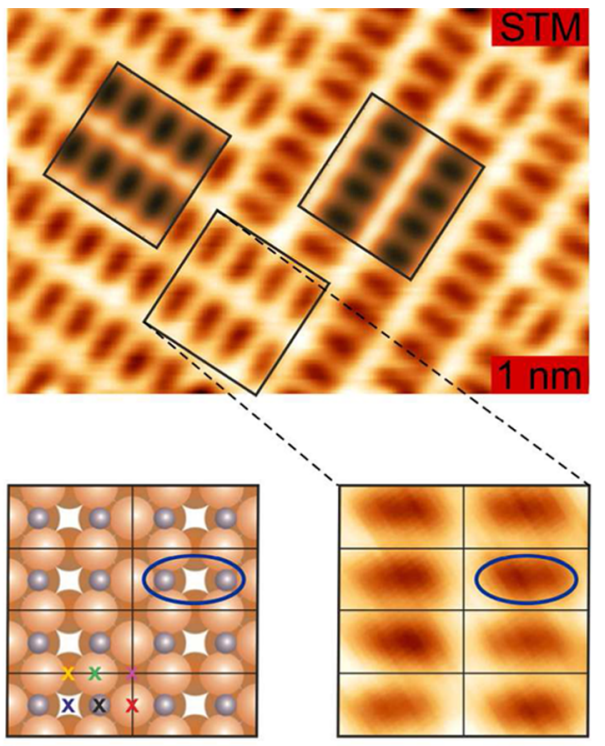

b)

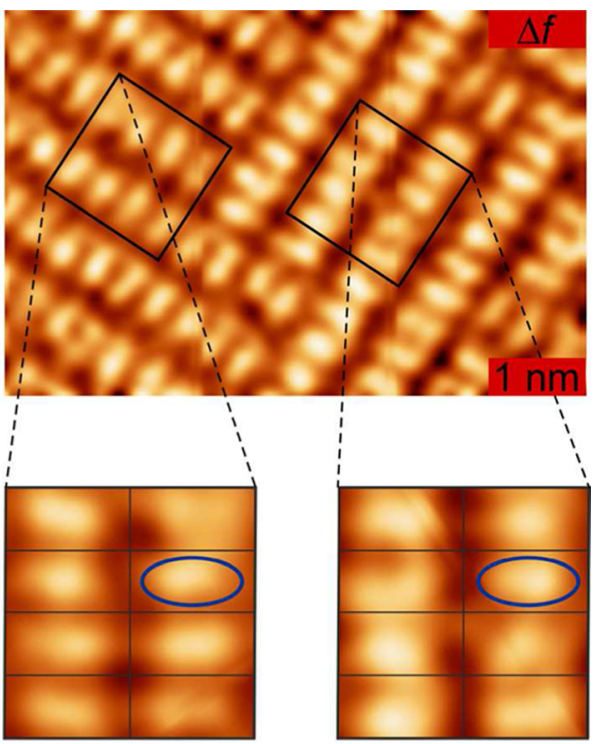

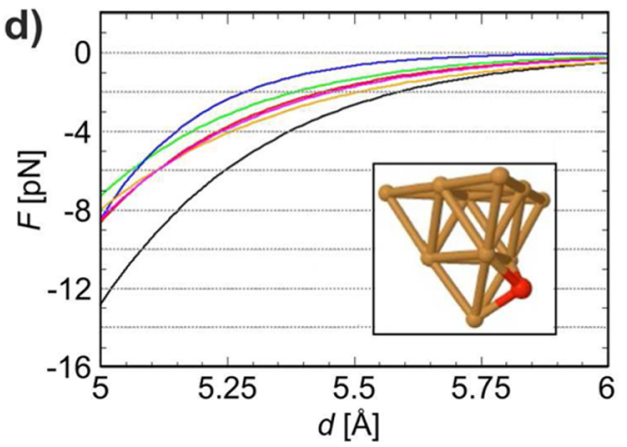

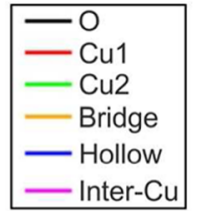

Figure 2. (a) Constant-current topography image obtained in dynamic STM mode showing two perpendicular structural domains $(U=-1.10 \mathrm{~V}$, $I_{\text {avg }}=400 \mathrm{pA}, T=60 \mathrm{~K}$ ). The two insets display theoretically calculated STM images for a Cu-CuO tip at the given experimental conditions. ${ }^{28}$ (b) Simultaneously acquired $\Delta f$ image featuring elongated bright spots. Two smaller regions of the acquired image corresponding to the locations of the insets in part (a) are highlighted. Please note that since for this tip the maximum tunneling current is expected to occur with an offset of about $1 \AA$ from the terminating copper atom in the direction of the oxygen atom (cf. inset in panel (d)), a lateral offset of that order between the positions of the actual lattice sites on the surface and the maxima imaged in STM has been taken into account. ${ }^{15}$ (c) Zoomed-in views of highlighted regions in parts (a) and (b), together with the corresponding surface model. Rectangular unit cells are depicted for reference. Smallest tunneling current values are recorded on the hollow sites between oxygen atoms on the missing rows, which are emphasized by blue ellipses, while the same regions are imaged the brightest in the frequency shift channel. (d) Calculated force interactions $\mathrm{F}$ between a $\mathrm{Cu}-\mathrm{CuO}$ tip apex as visualized in the inset and the six lattice sites labeled in Figure 1a plotted as a function of the tip-sample distance $d$. Negative values indicate attractive interactions.

oxides $^{32,33}$ are frequently employed in various chemical processes including NO reduction ${ }^{34}$ as well as CO oxidation, ${ }^{35}$ hence results regarding the local chemical reactivity of the surface oxide layer on $\mathrm{Cu}(100)$ as provided by combined STM/NC-AFM measurements would be particularly useful in elucidating the contribution of the catalyst surface to the associated phenomena on the atomic scale.

Despite the significant potential of combined STM/NCAFM measurements with regard to surface chemistry as discussed above, the results presented in this work point to a significant influence of topography-feedback-induced cross-talk on recorded $\Delta f$ data, preventing trivial conclusions to be made regarding atomic-scale chemical reactivity in such experiments. The associated discussion is concluded with a generalization to the case where multiple tip-sample interactions that show atomic-scale variations should be accurately measured.

\section{EXPERIMENTAL AND COMPUTATIONAL DETAILS}

A (100)-oriented copper single crystal has been cleaned by repeated cycles of $\mathrm{Ar}^{+}$sputtering and annealing under ultrahigh vacuum (UHV) conditions. Exposure of cleaned samples to 1000-3000 L molecular oxygen at an elevated temperature of $575 \mathrm{~K}$ then resulted in the formation of a surface oxide layer that is characterized by the $(2 \sqrt{ } 2 \times \sqrt{ } 2) R 45^{\circ}$ missing row reconstruction depicted in Figure 1a. Due to its $45^{\circ}$ orientation relative to the copper surface's closed-packed $\langle 011\rangle$ directions, two equivalent but perpendicular structural domains may form, which are frequently observed in the experiments. ${ }^{36}$ Combined STM/NC-AFM measurements in the dynamic STM mode (i.e., at constant average tunneling current) have been carried out using a home-built low-temperature microscope operating in UHV described in detail elsewhere. ${ }^{37}$ Electrochemically etched $\mathrm{Pt} / \mathrm{Ir}$ and $\mathrm{W}$ tips attached at the end of quartz tuning forks have been employed to collect the tunneling current and frequency shift data. ${ }^{38}$ All measurements have been performed at 
a)

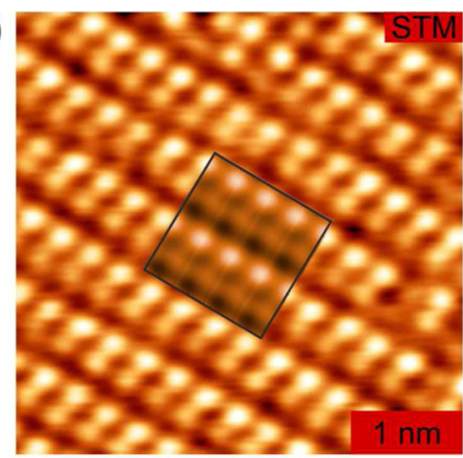

b)

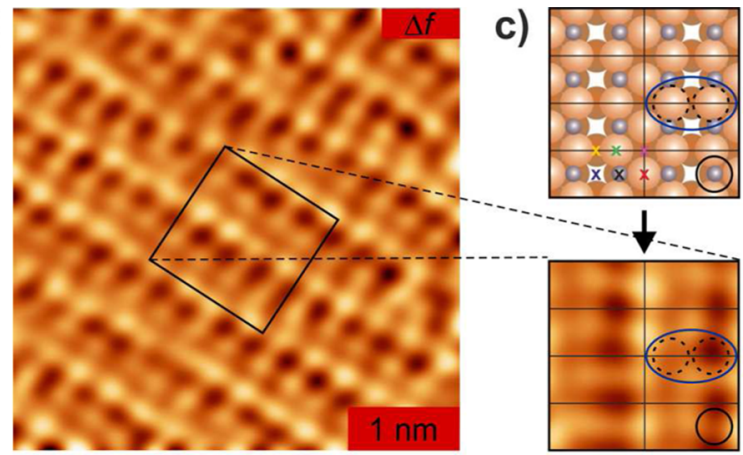

d)
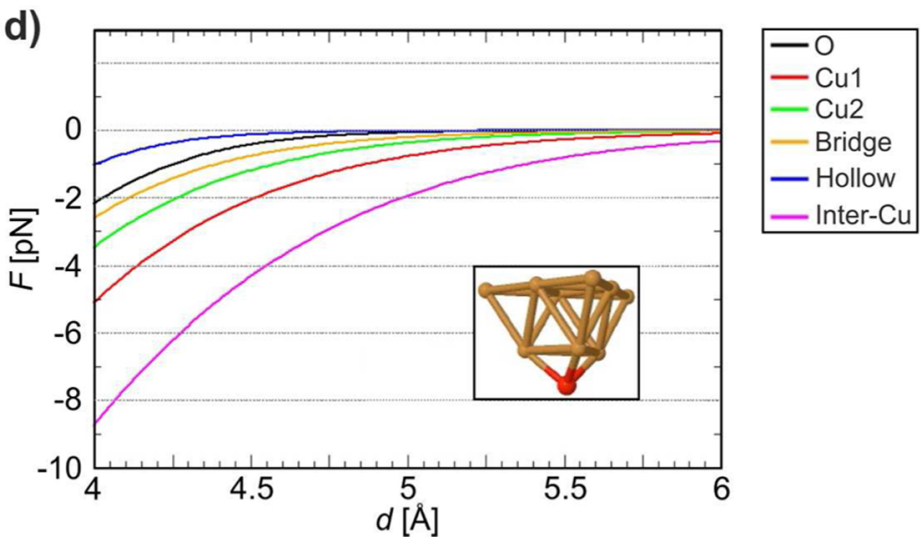

Figure 3. (a) Constant-current topography image obtained in dynamic STM mode ( $\left.U=-1.30 \mathrm{~V}, I_{\text {avg }}=500 \mathrm{pA}, T=60 \mathrm{~K}\right)$. The inset displays the theoretically calculated STM image for an oxygen-terminated model tip (cf. inset in panel (d) for a structural model) at the given experimental conditions. $^{28}$ (b) Simultaneously acquired $\Delta f$ image. A smaller region of the acquired image corresponding to the location of the inset in part (a) is highlighted. Please note that the asymmetric arrangement of the second-level copper atoms in the tip apex not only induces a slight $(\sim 1 \AA)$ lateral offset between the positions of the actual lattice sites on the surface and the maxima imaged in STM, but also distorts the image to a certain extent (i.e., it induces asymmetry into the image) ${ }^{28}$ (c) Zoomed-in view of the highlighted region in part (b), together with the corresponding surface model. Rectangular unit cells are depicted for reference. As discussed in detail in the main text, the two $\mathrm{Cu} 2$ atoms denoted by dashed black circles and highlighted by the blue ellipse display noticeable differences in interaction forces due to cross-talk. Cross-talk also makes $\mathrm{O}$ atoms such as the one denoted by the black circle display higher levels of interaction forces than adjacent $\mathrm{Cu} 2$ atoms even though the $\mathrm{Cu} 2$ sites interact stronger with the tip at equal distances (cf. panel (d)). (d) Calculated force interactions between an oxygen-terminated tip apex as illustrated by the structural model in the inset and the six lattice sites designated in Figure 1a as a function of tip-sample distance $d$. Based on calculation results, Inter-Cu sites are expected to exhibit the highest attractive interaction with the tip apex at constant tip-sample distances; however, the effect of cross-talk due to the tunneling current-based feedback leads to complications as discussed in the main text.

$T=60 \mathrm{~K}$ for improved stability and noise characteristics, with an oscillation amplitude of $5 \AA$.

Let us mention at this point that the term "cross-talk" is frequently used in the scanning probe microscopy community to refer to an electrical coupling between the tunneling current and frequency shift data channels during simultaneous STM/ NC-AFM measurements in certain tuning-fork based microscope designs as a result of stray capacitance, ${ }^{39}$ which is avoided when separate electrical connections for the collection of tunneling current and frequency shift are employed. ${ }^{37}$ As such, it should be emphasized that, in this work, we strictly refer to topography-feedback-induced cross-talk between data channels, rather than the specific electrical coupling issue mentioned above. Moreover, the "phantom force" effect has also been observed to result in a significant influence of the tunneling current on detected frequency shift values during simultaneous STM/NC-AFM measurements performed on samples with limited conductivity, for example, semiconductors, which is thus not relevant for our sample surface. ${ }^{40}$

The AFM calculations were performed using the VASP PAW method, with a $420 \mathrm{eV}$ cutoff and a $2 \times 2 \times 1 \mathrm{k}$-point mesh. To simulate tips coated with surface material, we utilized atomically sharp $\mathrm{Cu}(111)$ structures with an $\mathrm{O}$ atom contaminant $(\mathrm{Cu}-$ $\mathrm{CuO}$ tip) and $\mathrm{O}$ termination ( $\mathrm{CuO}$ tip). ${ }^{15,28}$ These three-layer AFM tip models were approached to the four-layer $\mathrm{Cu}-\mathrm{O}$ surface model (cell dimensions $14.6 \AA \times 14.6 \AA$ ) in steps of $0.25 \AA$ from the original height of $6 \AA$ to compute force spectroscopy curves. At each stage, the geometries of the two lower tip layers and two upper surface layers were optimized to a tolerance of $0.01 \mathrm{eV} / \AA$, while the rest of the atoms were constrained to simulate bulk regions extending into the probe and substrate. We employed a product of exponential and third power polynomial functions to fit theoretical force spectroscopy data points and produce smooth curves for straightforward data interpretation.

\section{RESULTS AND DISCUSSION}

The large-scale STM image in Figure $1 \mathrm{~b}$ shows a typical overview of the surface-oxidized $\mathrm{Cu}(100)$ sample. In agreement with previous STM studies, ${ }^{36,41}$ the resulting surface oxide layer is characterized by atomically flat terraces of $20-40 \mathrm{~nm}$ width 
separated by pinned, monatomic steps running predominantly in the $\langle 001\rangle$ directions of the $\mathrm{Cu}(100)$ substrate.

To obtain complementary information about the atomicscale structure of the surface as well as the interactions exhibited by individual lattice sites, combined STM/NC-AFM measurements have been performed on flat terraces of the reconstructed surface. As exemplified in Figures 2 and 3, different contrasts are observed for both the frequency shift and the constant-current STM topography channels, based on the use of different experimental parameters such as bias voltage $(U)$ and tunneling current $(I)$ as well as occasional in situ tip changes during experiments. Taking into account that tips have been treated by gentle crashes into the sample surface to achieve atomic resolution prior to recording each data set, it is reasonable to assume the respective apexes predominantly consist of copper and oxygen atoms. ${ }^{15}$ Based on ab initio simulations of STM images acquired on this sample surface using model tip apexes, one can readily identify the model tip structures responsible for each data set as well as the mean tipsample distance $d .^{28}$ From such a comparison, it is concluded that the ladder-type contrast exhibited by the STM image of Figure $2 \mathrm{a}$ at a bias voltage of $U=-1.10 \mathrm{~V}$ and a mean tipsample distance of $d=5.5 \AA$ was acquired with a $\mathrm{Cu}$ tip apex with an oxygen atom adsorbed on one side (the $\mathrm{Cu}-\mathrm{CuO}$ tip depicted in the inset of Figure $2 \mathrm{~d}$; see ref 28.). For both structural domains, the largest tunneling current was collected over the $\mathrm{Cu}$ atoms situated at the centers of filled rows $(\mathrm{Cu} 1$ atoms) forming bright stripes in the STM image, while bridges of smaller tunneling current are formed over $\mathrm{Cu} 2$ atoms situated next to the missing rows. On the other hand, the simultaneously recorded $\Delta f$ image (Figure $2 \mathrm{~b}$ ) is characterized by elongated bright spots (designating regions of apparently high attractive interaction with the probe tip). Carrying over the assignment of lattice sites from the STM image to the $\Delta f$ data, and taking into account the $\sim 1 \AA$ offset in lateral positions of actual atomic positions from the image maxima due to the inherent asymmetry of the tip apex, ${ }^{15,28}$ it can be determined that the elongated bright spots observed in the $\Delta f$ channel are centered between two oxygen atoms along the missing rows of the sample surface (Figure 2c). This particular assignment is at first glance consistent with $a b$ initio calculations of tip-sample interaction forces (Figure 2d), which indicate that surface oxygen exhibit the most attractive interaction with the $\mathrm{Cu}-\mathrm{CuO}$ model tip at all relevant tip-sample distances. If, however, the $\Delta f$ channel truly represents a map of the relative local interaction strength of surface sites, one would expect to resolve two individual maxima representing the two oxygen atoms. We will return below to why we observe an elongated bright spot on the hollow site instead.

Following a similar procedure for the data shown in Figure 3, it is determined that an oxygen-terminated copper tip apex (referred to as $\mathrm{CuO}$ tip; cf. the inset of Figure $3 \mathrm{~d}$ for a structural model) at a bias voltage of $U=-1.30 \mathrm{~V}$ and tipsample distance of $d=5.5 \AA$ closely reproduces the experimentally observed STM contrast (Figure 3a): The largest tunneling current is collected over $\mathrm{Cu} 2$ atoms on one side of the missing rows, while the $\mathrm{Cu} 2$ atoms on the other side of the missing rows appear significantly darker due to a particular asymmetry caused by the alignment of second layer $\mathrm{Cu}$ atoms of the tip apex model with respect to the surface. ${ }^{28}$ Additionally, taking into account that under these specific imaging conditions a lateral offset of $\sim 1 \AA$ between the actual positions of the $\mathrm{Cu}$ atoms and those imaged via STM has been predicted for the
$\mathrm{CuO}$ tip as well, ${ }^{28}$ one deduces from the $\Delta f$ channel that $\mathrm{Cu} 1$ atoms exhibit the largest attractive interaction with the probe tip (Figure 3b,c). This finding is, however, at odds with $a b$ initio simulations of the interactions for this tip apex model, which suggest that Inter-Cu sites should exhibit the greatest attractive forces with the apex (cf. Figure 3d). Moreover, it is observed that the experimental $\Delta f$ values measured for $\mathrm{Cu} 2$ atoms on both sides of the missing rows are significantly different despite the fact that the force interactions simulated on these two sites are virtually identical. Finally, $\mathrm{O}$ atoms seem to display a larger interaction with the probe tip compared to the $\mathrm{Cu} 2$ atoms in the experiment, which is again both counterintuitive and in conflict with the simulations.

The observed inconsistencies can, however, readily be explained by cross-talk between the data channels due to the active topography feedback: At locations that feature higher tunneling current, the tip moves away from the surface, thereby leading to a reduced $\Delta f$ at that spot due to an increase in tipsample distance. The inverse is true for locations of low tunneling current. As such, $\mathrm{O}$ atoms appear to interact more strongly with the tip apex than $\mathrm{Cu} 2$ atoms, despite the fact that the opposite would be true for measurements performed at a constant tip-sample distance. For the same reason, $\mathrm{Cu} 1$ sites appear more attractive than Inter- $\mathrm{Cu}$ sites and identical $\mathrm{Cu} 2$ sites seem to exhibit different chemical interaction forces.

Returning to the unanswered questions concerning the $\Delta f$ contrast in Figure 2, it should be noted that cross-talk causes the tip apex to be situated closer to the sample surface on top of hollow sites between $\mathrm{O}$ atoms on missing rows. This leads to a relative enhancement of the attractive interactions probed by the $\mathrm{Cu}$-terminated tip at those locations compared to the ideal case of constant-height imaging, which is why elongated bright spots are detected rather than individual bright spots for each $\mathrm{O}$ atom.

From the results presented here, it became evident that $\Delta f$ maps recorded in the dynamic STM mode are often affected by significant cross-talk effects associated with topography feedback and should therefore be viewed with great caution when evaluating atomic-scale surface interactions, if such an evaluation is possible at all. Consequently, the acquisition of three-dimensional data sets via $3 \mathrm{D}-\mathrm{AFM}$ and constant height imaging by, for example, disabling the feedback at low temperatures or by slowing the feedback down so it would not react to atomic-scale corrugations arise as the only viable and general methods of obtaining complementary information on site-specific chemical interaction forces and electronic structure without the influence of cross-talk.

While stable constant height imaging is mostly problematic on sample surfaces featuring steps, domain boundaries, and other defects as mentioned in the Introduction, 3D-AFM provides a robust technique with which reconstruction of true constant height tunneling current and frequency shift images can be accomplished. ${ }^{9}$ Moreover, active feedback control during data acquisition for 3D-AFM ensures stable imaging, even over surface irregularities. A comparison of experimentally obtained frequency shift and chemical interaction force maps using the dynamic STM and the 3D-AFM approaches, respectively, is presented in Figure 4 . While ab initio calculations allow determining the tip apex in both cases to be of the $\mathrm{Cu}-\mathrm{CuO}$ variety, the 3D-AFM method allows the unambiguous assignment of maximum chemical interaction forces to individual $\mathrm{O}$ atoms, whereas maximum interaction forces are erroneously assigned to the hollow sites between the 


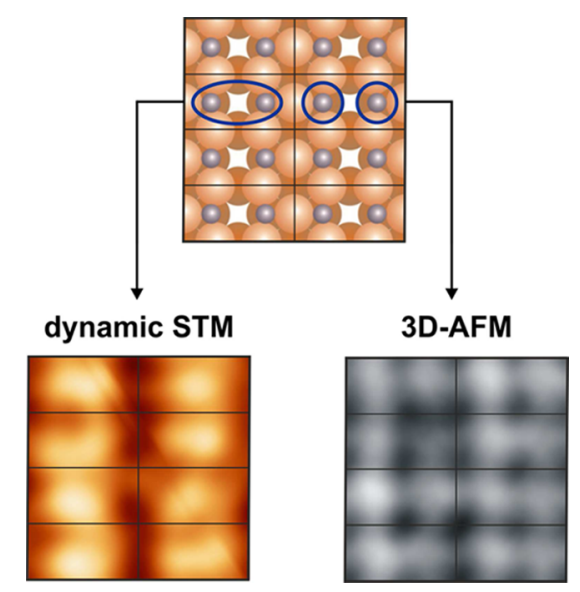

Figure 4. Comparison of atomic-scale chemical interaction measurements performed on the surface oxidized $\mathrm{Cu}(100)$ surface via the dynamic STM and 3D-AFM approaches. Please note that the frequency shift image corresponding to dynamic STM operation has been presented as the last inset of Figure 2c, while the map of chemical interaction forces acquired via 3D-AFM has been obtained from ref 15 . In both cases, the tip apex has been determined via ab initio calculations to be of the $\mathrm{Cu}-\mathrm{CuO}$ variety. As discussed earlier, the frequency shift data delivered by the dynamic STM method is affected by cross-talk and thus leads erroneously to the assignment of maximum chemical reactivity to the hollow sites between the oxygen atoms on the missing rows. On the other hand, constant height maps of chemical interaction forces extracted from 3D-AFM data result in the observation of single $\mathrm{O}$ atoms and thus allow the correct assignment of chemical interaction forces to specific atomic sites on the sample surface.

oxygen atoms in the dynamic STM mode due to the effect of cross-talk, as discussed earlier.

Let us note here that, in certain experimental investigations, a qualitative comparison of site-specific $\Delta f$ values can be sufficient to draw conclusions about atomic-scale surface properties to a first approximation, thus avoiding the need to perform an extensive quantification of interaction forces. In such cases, it should be sufficient to acquire only a small number of images at different frequency shift set points (corresponding to different mean tip-sample distances) than required for full three-dimensional data sets, as long as the data allow the construction of a single map of $\Delta f$ at a given constant height. On the other hand, if both constant height and 3DAFM operation are not possible, but stable operation in both STM and NC-AFM imaging modes can be separately achieved, reliable complementary information may only be obtained by imaging the surface region of interest alternatingly with both imaging modes, preferably at low temperatures to suppress the effect of lateral thermal drift.

Our findings can be generalized to the simultaneous acquisition of two physically independent interactions $\mathrm{A}$ and $\mathrm{B}$, while regulating on just one. The resulting information is stored in three different channels, where channels 1 and 2 record interactions $\mathrm{A}$ and $\mathrm{B}$, respectively, and channel 3 contains the $z$ signal (i.e., the "topography") produced by the feedback attempting to keep interaction A constant. If the $z$ corrugation in channel 3 is comparable to or larger than the decay length of interaction $B$, it is not possible to obtain useful information about interaction B. In this case, any assignment of image features observed in channel 2 to lattice sites will be risky at best, in particular if structural asymmetries at the tip apex induce an offset between channels 2 and 3 , as we have found to be commonly the case. The situation only changes if the corrugation in channel 3 is lower (ideally much lower) than the typical decay length of interaction $B$ so that the images obtained in channel 2 can be considered to be more or less constant height. This specific case may occur if interactions A and B feature greatly different decay lengths and one chooses the interaction with the shorter decay length as the feedback signal. Alternatively, as pointed out earlier, one can slow the feedback loop to a degree that atomic-scale features are not followed. In this case, "quasi-constant-height" imaging is achieved with physically relevant information in channels 1 and 2.

Let us conclude the discussion presented in this section by mentioning that the contrasts observed for both data channels might also be influenced by structural asymmetry of the probe tip on a larger scale than the model apexes considered here, as well as by other complications such as elastic deformations. ${ }^{42}$ Consequently, it is not always possible to perform straightforward assignments of atomic positions to specific features in data sets that show strong asymmetric features in both data channels.

\section{CONCLUSIONS}

Combined STM/NC-AFM data sets simultaneously recorded in the dynamic STM mode on surface-oxidized $\mathrm{Cu}(100)$ have been presented in conjunction with ab initio simulations of force interactions for model tip apexes with the aim of testing to what degree atomic-scale surface interactions can be characterized locally using individual images featuring $\Delta f$ data. The results suggest that recorded frequency shift data are often strongly influenced by cross-talk due to active topography feedback, leading to potentially erroneous interpretations of atomic-scale interactions on surfaces. To avoid such complications, we recommend performing constant-height measurements of frequency shift and tunneling current or acquiring three-dimensional data sets provided using the 3D-AFM approach.

\section{AUTHOR INFORMATION}

\section{Corresponding Authors}

*E-mail: mehmet.baykara@bilkent.edu.tr.

*E-mail: udo.schwarz@yale.edu.

\section{Author Contributions}

The manuscript was written through contributions of all authors. All authors have given approval to the final version of the manuscript.

\section{Notes}

The authors declare no competing financial interest.

\section{ACKNOWLEDGMENTS}

Financial support from the National Science Foundation through the Yale Materials Research Science and Engineering Center (Grant No. MRSEC DMR-1119826), the U.S. Department of Energy (Basic Energy Sciences Grant No. DE-FG0206ER15834), the Spanish MINECO (Projects MAT200802953-E, MAT2011-023627, and CSD2010-00024), and from the UAM-Banco Santander Program of Collaboration with the United States is gratefully acknowledged. Access to the Magerit Supercomputer (CesViMa, Madrid) was provided through the Spanish Supercomputing Network (RES, Spain). H.M. acknowledges support by the Deutsche Forschungsgemeinschaft through the transregional collaborative research center 
TRR 061 (Project B7). M.Z.B. acknowledges support by the Outstanding Young Scientist program of the Turkish Academy of Sciences (TÜBA-GEBIP).

\section{REFERENCES}

(1) Geim, A. K. Graphene: Status and Prospects. Science 2009, 324, $1530-1534$.

(2) Altman, E. I.; Schwarz, U. D. Structural and Electronic Heterogeniety of Two Dimensional Amorphous Silica Layers. Adv. Mater. Interfaces 2014, 1, 1400108.

(3) Bonnell, D. A.; Basov, D. N.; Bode, M.; Diebold, U.; Kalinin, S. V.; Madhavan, V.; Novotny, L.; Salmeron, M.; Schwarz, U. D.; Weiss, P. S. Imaging Physical Phenomena with Local Probes: From Electrons to Photons. Rev. Mod. Phys. 2012, 84, 1343-1381.

(4) Chen, C. J. Introduction to Scanning Tunneling Microscopy; Oxford University Press: Oxford, U.K., 2007.

(5) Morita, S.; Wiesendanger, R.; Meyer, E. Noncontact Atomic Force Microscopy; Springer: Berlin, Germany, 2002.

(6) Hölscher, H.; Schwarz, A.; Allers, W.; Schwarz, U. D.; Wiesendanger, R. Quantitative Analysis of Dynamic-Force-Spectroscopy Data on Graphite(0001) in the Contact and Noncontact Regimes. Phys. Rev. B 2000, 61, 12678-12681.

(7) Lantz, M. A.; Hug, H. J.; Hoffmann, R.; van Schendel, P. J. A.; Kappenberger, P.; Martin, S.; Baratoff, A.; Guntherodt, H. J. Quantitative Measurement of Short-Range Chemical Bonding Forces. Science 2001, 291, 2580-2583.

(8) Sugimoto, Y.; Pou, P.; Abe, M.; Jelinek, P.; Perez, R.; Morita, S.; Custance, O. Chemical Identification of Individual Surface Atoms by Atomic Force Microscopy. Nature 2007, 446, 64-67.

(9) Baykara, M. Z.; Schwendemann, T. C.; Altman, E. I.; Schwarz, U. D. Three-Dimensional Atomic Force Microscopy - Taking Surface Imaging to the Next Level. Adv. Mater. 2010, 22, 2838-2853.

(10) Albers, B. J.; Schwendemann, T. C.; Baykara, M. Z.; Pilet, N.; Liebmann, M.; Altman, E. I.; Schwarz, U. D. Three-Dimensional Imaging of Short-Range Chemical Forces with Picometre Resolution. Nat. Nanotechnol. 2009, 4, 307-310.

(11) Baykara, M. Z.; Schwendemann, T. C.; Albers, B. J.; Pilet, N.; Mönig, H.; Altman, E. I.; Schwarz, U. D. Exploring Atomic-Scale Lateral Forces in the Attractive Regime: A Case Study on Graphite (0001). Nanotechnology 2012, 23, 405703.

(12) Gross, L.; Mohn, F.; Moll, N.; Liljeroth, P.; Meyer, G. The Chemical Structure of a Molecule Resolved by Atomic Force Microscopy. Science 2009, 325, 1110-1114.

(13) Sweetman, A. M.; Jarvis, S. P.; Sang, H.; Lekkas, I.; Rahe, P.; Wang, Y.; Wang, J.; Champness, N. R.; Kantorovich, L.; Moriarty, P. Mapping the Force Field of a Hydrogen-Bonded Assembly. Nat. Commun. 2014, 5, 3931.

(14) Welker, J.; Giessibl, F. J. Revealing the Angular Symmetry of Chemical Bonds by Atomic Force Microscopy. Science 2012, 336, 444-449.

(15) Baykara, M. Z.; Todorović, M.; Mönig, H.; Schwendemann, T. C.; Unverdi, O.; Rodrigo, L.; Altman, E. I.; Perez, R.; Schwarz, U. D. Atom-Specific Forces and Defect Identification on Surface-Oxidized $\mathrm{Cu}(100)$ with Combined 3D-AFM and STM Measurements. Phys. Rev. B 2013, 87, 155414.

(16) Albers, B. J.; Schwendemann, T. C.; Baykara, M. Z.; Pilet, N.; Liebmann, M.; Altman, E. I.; Schwarz, U. D. Data acquisition and Analysis Procedures for High-Resolution Atomic Force Microscopy in Three Dimensions. Nanotechnology 2009, 20, 264002.

(17) Baykara, M. Z.; Dagdeviren, O. E.; Schwendemann, T. C.; Mönig, H.; Altman, E. I.; Schwarz, U. D. Probing Three-Dimensional Surface Force Fields with Atomic Resolution: Measurement Strategies, Limitations, and Artifact Reduction. Beilstein J. Nanotechnol. 2012, 3, 637-650.

(18) Enevoldsen, G. H.; Pinto, H. P.; Foster, A. S.; Jensen, M. C. R.; Kuhnle, A.; Reichling, M.; Hofer, W. A.; Lauritsen, J. V.; Besenbacher, F. Detailed Scanning Probe Microscopy Tip Models Determined from
Simultaneous Atom-Resolved AFM and STM Studies of the $\mathrm{TiO}(2)$ (110) Surface. Phys. Rev. B 2008, 78, 045416.

(19) Sweetman, A.; Stannard, A.; Sugimoto, Y.; Abe, M.; Morita, S.; Moriarty, P. Simultaneous Noncontact AFM and STM of Ag:Si(111)$(\sqrt{3} \times \sqrt{3})$ R30. Phys. Rev. B 2013, 87, 075310.

(20) Guggisberg, M.; Bammerlin, M.; Luthi, R.; Loppacher, C.; Battiston, F.; Lu, J.; Baratoff, A.; Meyer, E.; Guntherodt, H. J. Comparison of Dynamic Lever STM and Noncontact AFM. Appl. Phys. A: Mater. Sci. Process. 1998, 66, S245-S248.

(21) Ozer, H. O.; Norris, A.; Oral, A.; Hoffmann, P. M.; Pethica, J. B. Low-Amplitude, Force Gradient Imaging of $\mathrm{Cu}(100)$ Surface Using Tunnel Current Feedback. Nanotechnology 2004, 15, S5-S8.

(22) Resta, A.; Leoni, T.; Barth, C.; Ranguis, A.; Becker, C.; Bruhn, T.; Vogt, P.; Le Lay, G. Atomic Structures of Silicene Layers Grown on $\mathrm{Ag}(111)$ : Scanning Tunneling Microscopy and Noncontact Atomic Force Microscopy Observations. Sci. Rep. 2013, 3, 2399.

(23) Hembacher, S.; Giessibl, F. J.; Mannhart, J.; Quate, C. F. Local Spectroscopy and Atomic Imaging of Tunneling Current, Forces, and Dissipation on Graphite. Phys. Rev. Lett. 2005, 94, 056101.

(24) Sawada, D.; Sugimoto, Y.; Morita, K.; Abe, M.; Morita, S. Simultaneous Measurement of Force and Tunneling Current at Room Temperature. Appl. Phys. Lett. 2009, 94, 173117.

(25) Majzik, Z.; Tchalala, M. R.; Svec, M.; Hapala, P.; Enriquez, H.; Kara, A.; Mayne, A. J.; Dujardin, G.; Jelinek, P.; Oughaddou, H. Combined AFM and STM Measurements of a Silicene Sheet Grown on the $\operatorname{Ag}(111)$ Surface. J. Phys.: Condens. Matter 2013, 25, 225301.

(26) Majzik, Z.; Drevniok, B.; Kaminski, W.; Ondracek, M.; McLean, A. B.; Jelinek, P. Room Temperature Discrimination of Adsorbed Molecules and Attachment Sites on the Si(111) $7 \times 7$ Surface Using a qPlus Sensor. ACS Nano 2013, 7, 2686-2692.

(27) Altman, E. I.; Schwarz, U. D. Mechanisms, Kinetics, and Dynamics of Oxidation and Reactions on Oxide Surfaces Investigated by Scanning Probe Microscopy. Adv. Mater. 2010, 22, 2854-2869.

(28) Mönig, H.; Todorović, M.; Baykara, M. Z.; Schwendemann, T. C.; Rodrigo, L.; Altman, E. I.; Perez, R.; Schwarz, U. D. Understanding Scanning Tunneling Microscopy Contrast Mechanisms on Metal Oxides: A Case Study. ACS Nano 2013, 7, 10233-10244.

(29) Vang, R. T.; Lauritsen, J. V.; Laegsgaard, E.; Besenbacher, F. Scanning Tunneling Microscopy as a Tool to Study Catalytically Relevant Model Systems. Chem. Soc. Rev. 2008, 37, 2191-2203.

(30) Longwitz, S. R.; Schnadt, J.; Vestergaard, E. K.; Vang, R. T.; Laegsgaard, E.; Stensgaard, I.; Brune, H.; Besenbacher, F. HighCoverage Structures of Carbon Monoxide Adsorbed on Pt (111) Studied by High-Pressure Scanning Tunneling Microscopy. J. Phys. Chem. B 2004, 108, 14497-14502.

(31) Bennett, R. A.; Pang, C. L.; Perkins, N.; Smith, R. D.; Morrall, P.; Kvon, R. I.; Bowker, M. Surface Structures in the SMSI State; Pd on $(1 \times 2)$ reconstructed $\mathrm{TiO}_{2}$ (110). J. Phys. Chem. B 2002, 106, $4688-4696$.

(32) Ciston, J.; Si, R.; Rodriguez, J. A.; Hanson, J. C.; Martinez-Arias, A.; Fernandez-Garcia, M.; Zhu, Y. Morphological and Structural Changes during the Reduction and Reoxidation of $\mathrm{CuO} / \mathrm{CeO} 2$ and $\mathrm{Ce}_{1-x} \mathrm{Cu}_{x} \mathrm{O}_{2}$ Nanocatalysts: In Situ Studies with Environmental TEM, XRD, and XAS. J. Phys. Chem. C 2011, 115, 13851-13859.

(33) Martínez-Arias, A.; Hungría, A. B.; Fernandez-García, M.; Conesa, J. C.; Munuera, G. Interfacial Redox Processes under $\mathrm{CO} / \mathrm{O}_{2}$ in a Nanoceria-Supported Copper Oxide Catalyst. J. Phys. Chem. B 2004, 108, 17983-17991.

(34) Blanco, J.; Garcia de la Banda, J. F.; Avila, P.; Melo, F. Selective Reduction of Nitric Oxide on Nickel Oxide-Copper Oxide Supported Catalysts. J. Phys. Chem. 1986, 90, 4789-4793.

(35) Wang, Y.; Wu, G.; Yang, M.; Wang, J. Competition between Eley-Rideal and Langmuir-Hinshelwood Pathways of CO Oxidation on $\mathrm{Cu}_{n}$ and $\mathrm{Cu}_{n} \mathrm{O}(n=6,7)$ Clusters. J. Phys. Chem. C 2013, 117, $8767-8773$

(36) Leibsle, F. M. STM Studies of Oxygen-Induced Structures and Nitrogen Coadsorption on the $\mathrm{Cu}(100)$ Surface-Evidence for a OneDimensional Oxygen Reconstruction and Reconstructive Interactions. Surf. Sci. 1995, 337, 51-66. 
(37) Albers, B. J.; Liebmann, M.; Schwendemann, T. C.; Baykara, M. Z.; Heyde, M.; Salmeron, M.; Altman, E. I.; Schwarz, U. D. Combined Low-Temperature Scanning Tunneling/Atomic Force Microscope for Atomic Resolution Imaging and Site-Specific Force Spectroscopy. Rev. Sci. Instrum. 2008, 79, 033704.

(38) Giessibl, F. J. High-Speed Force Sensor for Force Microscopy and Profilometry Utilizing a Quartz Tuning Fork. Appl. Phys. Lett. 1998, 73, 3956-3958.

(39) Majzik, Z.; Setvin, M.; Bettac, A.; Feltz, A.; Chab, V.; Jelinek, P. Simultaneous Current, Force and Dissipation Measurements on the $\mathrm{Si}(111) 7 \times 7$ Surface with an Optimized qPlus AFM/STM Technique. Beilstein J. Nanotechnol. 2012, 3, 249-259.

(40) Weymouth, A. J.; Wutscher, T.; Welker, J.; Hofmann, T.; Giessibl, F. J. Phantom Force Induced by Tunneling Current: A Characterization on $\mathrm{Si}(111)$. Phys. Rev. Lett. 2011, 106, 226801.

(41) Jensen, F.; Besenbacher, F.; Laegsgaard, E.; Stensgaard, I. Dynamics of Oxygen-Induced Reconstruction of $\mathrm{Cu}(100)$ Studied by Scanning Tunneling Microscopy. Phys. Rev. B 1990, 42, 9206-9209. (42) Uluutku, B.; Baykara, M. Z. Effect of Lateral Tip Stiffness on Atomic-Resolution Force Field Spectroscopy. J. Vac. Sci. Technol., B 2013, 31, 041801. 